\title{
Lexical networks
} between sounds and meanings in Taiwan Mandarin: Evidence from psycholinguistics

\begin{abstract}
The present study aims to provide an outline of lexical networks between sounds and meanings in Taiwan Mandarin. Both the phonological and semantic relations are explored by a free word association task. Previous research has demonstrated a significant influence of phonology on Indo-European networks and suggested the influence of semantics on languages with lexicography such as Mandarin. With a new methodology in which word frequency, syllable type, syllable structure, tone structure, imageability, and parts of speech are carefully considered for the experimental stimuli, a total of 248 responses were collected from six Taiwan Mandarin native speakers. The results of lexical associations suggest that: 1) semantic relations show a stronger connection than phonological ones in Taiwan Mandarin networks; 2) rhymes present a stronger influence on associations than initial segments. The understanding of lexical organization in the human brain may contribute to further research on functions of associations and networks between human and artificial intelligence.
\end{abstract}

Keywords: lexical networks, lexical associations, semantic-phonological organization, Taiwan Mandarin.

\section{Introduction}

Lexical networks represent the organization of human lexicon which functions differently regarding individual and regional variabilities as well as the constraints of languages. Several methodologies have so far been implemented to explore the connections and organizations within lexical networks, such as Stroop, lexical decision, word categorization, recognition memory, lexical association tasks, and speech errors. Through lexical associations, the mapping and decoding process between formed concepts during perception and production can be traced by the relations between target words and responded words. For example, the ways the associated word pairs 'peach' and 'pear' in English, and 儲存 'to save' [ts ${ }^{\mathrm{h}} \mathrm{u} 35 \mathrm{ts}{ }^{\mathrm{h}}$ wən35] and 刪除 'to delete' [san55ts ${ }^{\mathrm{h}} \mathrm{u} 35$ ]

1 Address for correspondence: Graduate Institute of Linguistics, National Chengchi University, Taipei, 11605 Taiwan. E-mail: ttseng@g.nccu.edu.tw 
in Mandarin connect to each other can probably provide a clue of the organization of lexical networks. The lexical organization in a language can possibly be influenced by similar or identical sounds, meanings, parts of speech, etc. Therefore, approaching the lexical organization in the human mind can help with the understanding of the retrieval, comprehension, acquisition and usage of the mental lexicon.

Previous works in the related fields have proposed some widely accepted theories and assumptions concerning lexical networks. For example, in Indo-European languages, there is evidence of the phonological similarity, especially the initial segments, within the connections (e.g., Dell et al. 2000; Vitevitch 2008; Vitevitch \& Goldstein 2014; Beckage \& Colunga 2016). On the other hand, the semantic similarity in the Mandarin lexicon in terms of various semantic relations such as paradigmatic and syntagmatic ones, have been suggested by a few works (e.g., Huang \& Hsieh 2015; Hsieh 2006, 2016; Wan \& Ting 2019; Tang \& Wan 2020). However, the target issue has not been widely researched in the Mandarin lexicon. Most of the studies do not consider adequate linguistic features. The objectives of the present paper are therefore to fill this research gap as well as to investigate how the phonological and semantic influences function and interact within the lexicon. To further explore the lexical networks in the aspects of the relations between sounds and meanings in Taiwan Mandarin, a free word association task was conducted with disyllabic nouns and verbs. During the lexical association task, the participants were required to produce the first word emerging in their minds upon a lexical item as the stimulus. The analyses and the categorizations of the responses of the free word association tasks later focused on the interaction between phonology and semantics.

\section{Lexical networks}

\subsection{Influences on connections}

Several influences have been attested to frame the structure of lexical networks, including frequency, imageability, lexical bias, part of speech, and semantic as well as phonological factors (e.g., Reeves et al. 1998). Besides, an individual's language experience, culture and/or regional diversity can also affect a word's frequency by using it in diverse contexts. A number of studies of each factor have been conducted. For example, in Dell's (1990) spreading activation model of lexical retrieval, frequency is the key factor to affect phonological errors. Kittredge et al. (2008) also find evidence from aphasic picture naming in which lexical frequency has significant influences on both phonological and semantic errors. Imageability indicates the concreteness and abstractness of a concept. An internal image could be more easily generated by processing words with concrete concepts, such as book, than the abstract ones, for instance, peace. Rich semantic representation encoded in words with higher imageability is attested to be 
the reason for easier access and deeper storage in the brain. De Groot (1989) finds that imageability strongly determines responses in word associations, and Caplan \& Madan (2016) propose that imageability is one of the most important factors involved in language processing. Furthermore, during the lexical access, the tendency to produce a real word instead of a novel word, i.e., lexical bias, has been supported by previous studies (e.g., Dell 1985; Dell \& O’Seaghdha's 1991, 1992). Different parts of speech determine the functions of a word in various contexts and the connections between semantic and syntactic relations. For instance, both Nissen \& Henriksen's (2006) and De Simone \& Collina's (2016) evidence support the claim that parts of speech influence the responses during lexical access and production within lexical networks.

\subsection{Indo-European networks}

Phonologically related factors, another integral basis of language network representations, have been identified by a large number of studies in Indo-European languages (e.g., Dell et al. 2000; Vitevitch 2008; Vitevitch \& Goldstein 2014; Beckage \& Colunga 2016). In Dell et al.'s (2000) research about speech errors, the scholars find that although speech errors concerning novel words happen often, the tendency of phonotactic violations is relatively low. Another major phonological influence is initialness, i.e., onsets tend to be preserved during the process of lexical access and retrieval. For instance, Vitevitch (2008) presents a small portion of the English phonological network by 20,000 English words to represent word speech and neighbors of speech shown as Figure 1.

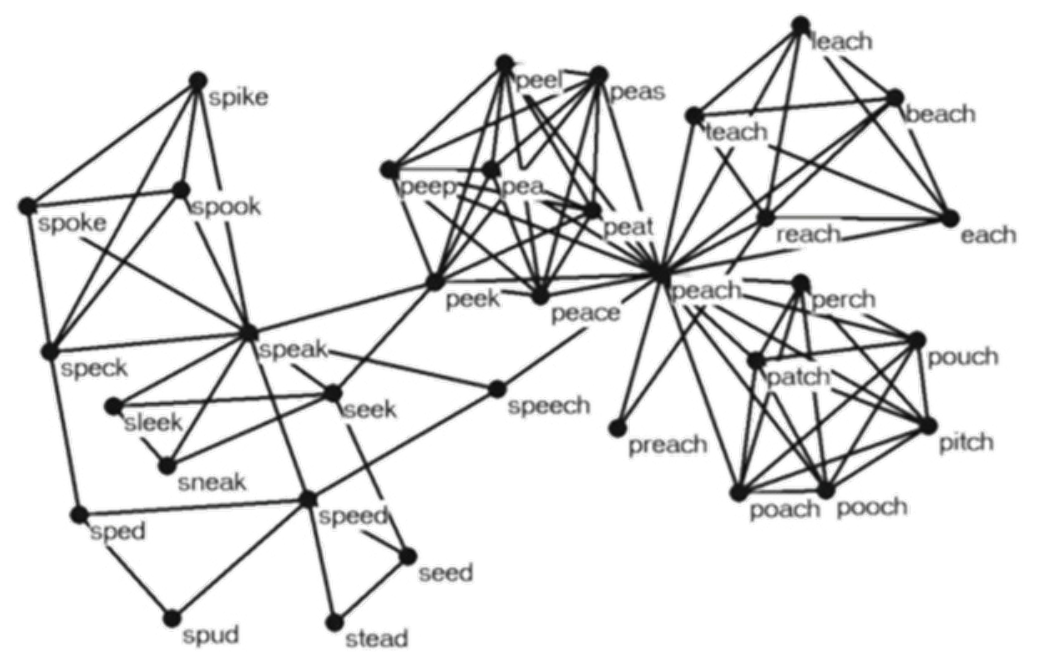

Figure 1. A sample of English phonological networks analyzed in Vitevitch (2008)

The figure illustrates the phonological networks including the phenomena of initialness, in which the nodes represent words that are connected by links within the networks. The core of this example, the node for the word peach [pit]] has a link connecting to other representations such as preach [p.itf], peat [pit], patch [pæt]], each [it]], etc. 
The example shows the phonological similarity of words with [p] as the identical onset, and the word peach [pitf] is directly connected to its minimal pair pea [pi]; however, it is indirectly connected to other words, such as speak [spik] and speed [spid]. This is also further explained by Beckage \& Colunga (2016) as "edit distance", i.e., a series of phoneme changes required to transfer one word into another. For example, the word kit can substitute its onset into [h] for another word hit; insert another consonant [s] in the word-initial to constitute the consonant cluster [sk] for the word skit; or delete the onset $[\mathrm{k}]$ and remaining the rhyme to form another word $i t$. Therefore, word edited with more than one step from the target word would need more nodes to indirectly connect with others within the networks.

\subsection{Mandarin networks}

On the other hand, different linguistic constraints may alter the lexical organization. Regarding the strong dependence of Mandarin lexicon on its morpho-semantic system, a few of models and theories have illustrated the unique lexical networks in Mandarin (e.g., Huang \& Hsieh 2015; Hsieh 2006, 2016). Based on the idea of charactercentered lexical networks in Mandarin, Huang \& Hsieh (2015) review existing studies and propose that Mandarin lexicon is encoded with the conventionalized semantic as well as morpho-syntactic information by its orthographic system. A pyramid structure model schematizes this viewpoint by a three-leveled representation with nodes and links connecting the concepts in Mandarin as Figure 2.

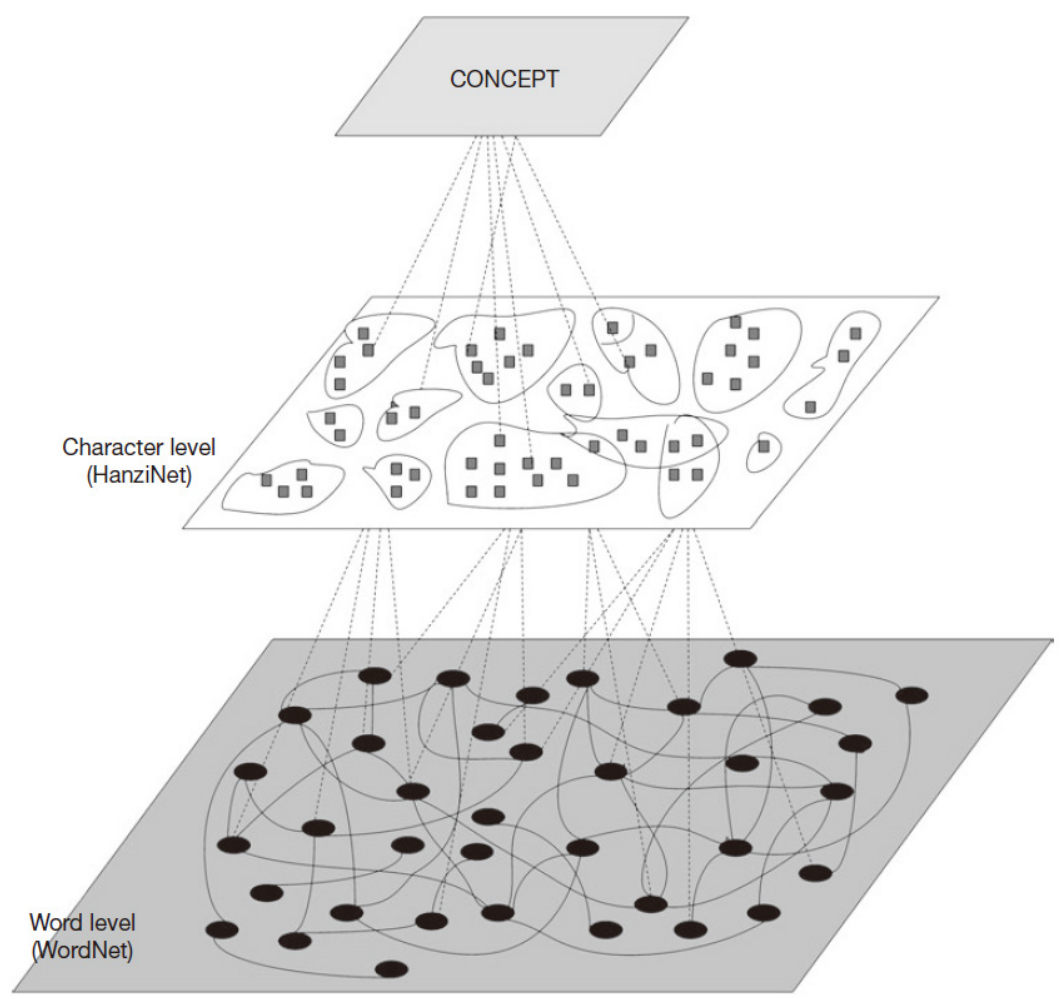

Figure 2. The pyramid structure model in Hsieh $(2006,2016)$ 
This model proposed by Hsieh (2006, 2016) demonstrates the encoding of the lexicography between the process from message to lexical representations. The interactions between characters and morphemes operate in the character level. In this level, similar information groups as a cluster to map conceptual organizations into lexical representations. In the word level, words are connected to each other via different semantic relations in Mandarin lexical networks. That is to say, rich sematic information encoded in both characters and words in Mandarin makes its lexical networks function differently against the languages without this system.

Related studies of the Mandarin lexicon have so far been researched mainly with insufficient linguistic features included. Most of them focus on bilingual comparison in Beijing Mandarin (e.g., Lee 2012; Zhao 2013). Several studies are based on the theory of remote association test by Mednick (1962) to build creativity assessments (e.g., Huang et al. 2012; Lee 2014; Chang et al. 2016). The other of them, related to associations, mostly do not consider phonological features in Mandarin for experimental materials. The relations between targets and responses as well as the comparison between semantic and phonological encodings are not discussed.

\subsection{Research questions and hypotheses}

Considering the crucial influence of initialness in Indo-European networks and the strong encodings of lexical semantics in Mandarin, the question about the tendency of lexical organization in Mandarin, a tone language with a lexicographical system, has arisen. To investigate the organization of lexical networks between sounds and meanings in Taiwan Mandarin, the linguistic features such semantic and phonological factors should first be considered as foundations. Later, the idea should be supported by experimental evidence. However, most of the research related to Mandarin lexicon focuses on bilingual comparison (e.g., Lee 2012; Zhao 2013), or analyzes the responses to associations without linguistic features in Mandarin considered as the basis. Therefore, the possible influences on Mandarin lexicon such as word frequency, syllable types, syllable structures, tone structures, imageability and syntactic categories are all considered as the experimental foundations in the present study to fill this gap.

The present study therefore aims to provide an outline of the psychological networks between sounds and meanings in Taiwan Mandarin by free lexical associations with 42 disyllabic nouns and verbs as the targets. The topics to be investigated include the following: 1) the phonological influences in terms of initialness in Taiwan Mandarin; 2) the semantic influences on Taiwan Mandarin networks; 3) the interactions between these two influences in Taiwan Mandarin.

Compared to the semantic relations, the phonological relations are predicted to present weaker connections between stimuli and responses, and the rhymes instead of the initial consonants may probably show more influences due to the related encoding 
systems in Mandarin. Regarding the previous works on the Mandarin lexicon and lexical associations (e.g., Zhao 2013; Huang \& Hsieh 2015; Hsieh 2006, 2016; Zhang \& Chen 2018; Tang \& Wan 2020), semantic encoding is expected to show stronger connections than phonology in the lexical networks of Taiwan Mandarin. Within the semantic relations, the paradigmatic relation is presumed to dominate in the responses to noun targets as in the previous studies (e.g., Nissen \& Henriksen 2006; Zhao 2013; Zhang \& Chen 2018; Wan \& Ting 2019). Moreover, the interactions between the semantic and phonological relations of the responses are expected to present a high proportion of semantic relation. This prediction results not only from the rich semantic information encoded in both characters and words in Mandarin (see Hsieh 2006, 2016 for more information), but also from the research methodology by lexical association tasks in the present study.

\section{Lexical association tasks}

Lexical association is assumed to reflect fundamental connections within lexical networks. It allows the concepts to be free from the use of pragmatics for extended purposes, or breaks out of the frame of syntax as a sentence. That is to say, lexical association is believed to be a simple reflection of what prominently comes to mind. This is why it is used in the present study.

\subsection{Participants, materials and procedures}

To explore the lexical organization in Taiwan Mandarin, six Taiwan Mandarin native speakers (age mean=24, $\mathrm{SD}=2.45 ; 3$ males and 3 females) without any explicit training in linguistics were included in the lexical association tasks. The experimental material contained 42 Mandarin disyllabic words including common nouns ( $\mathrm{N}=21)$ and activity transitive verbs $(\mathrm{N}=21)^{2}$, which are tagged with the highest frequency in Academic Sinica Balanced Corpus 3.0 (Sinica Corpus). This corpus was created by Huang et al. (1995) and maintained by Chinese Knowledge and Information Processing Group (CKIP 1998) ${ }^{3}$. All the Taiwan Mandarin onsets $(\mathrm{N}=21)$, syllable structures $(\mathrm{N}=4)$, and tone structures $(\mathrm{N}=4)$ of the target words were counterbalanced with the word frequency, syntactic categories, and imageability considered. The onsets were randomly arranged into the two syllables of the 42 target words $[\mathrm{p}],\left[\mathrm{p}^{\mathrm{h}}\right],[\mathrm{t}],\left[\mathrm{t}^{\mathrm{h}}\right],[\mathrm{k}],\left[\mathrm{k}^{\mathrm{h}}\right],[\mathrm{m}],[\mathrm{n}],[1],[\mathrm{f}],[\mathrm{x}],[\mathrm{t} 6],\left[\mathrm{t}^{\mathrm{h}}\right],[6],[\mathrm{ts}],\left[\mathrm{ts}{ }^{\mathrm{h}}\right]$, $[\mathrm{s}],[\mathrm{ts}],\left[\mathrm{ts}_{\mathrm{h}}^{\mathrm{h}}\right],[\mathrm{s}]$, and [z]. In other words, each possible onset presented only once in the

2 The selection of stimuli's lexical categories does not include adjectives because both nouns and verbs can be modifiers in Taiwan Mandarin. Further discussions of the related syntactic issues are not included in the present study.

3 The Academic Sinica Balanced Corpus 3.0 (Sinica Corpus) contains five million words with a userfriendly search interface (http://elearning.ling.sinica.edu.tw/CWordfreq_index.html). It is also the first fully POS-tagged balanced Chinese Corpus including 46 different tags. 
first syllable and once in the second syllable of the 21 nouns, and the same arrangement was done for the 21 verbs. The criterion of the syllable structures was according to Duanmu's (1990, 2000) CVX theory. The word frequency and parts-of-speech tagging were referred from Sinica Corpus; imageability was determined by the highest category of the target words as physical, abstract or state in E-HowNet (CKIP 2009). The participants could move on to the following procedures only if they understood the objectives of the tasks as well as agreed with the data and information collection. The experiment was conducted by E-Prime 3.0 evaluation version, after a five-trail practice stage for the participants first with a question section followed, the formal task presented 42 target words randomly on a laptop screen. With one visual target primed at once, the participants were requested to give an oral response, i.e., a Mandarin word associating to the target immediately.

\subsection{Data analysis and associations}

Since this study investigates lexical networks between sounds and meanings in Taiwan Mandarin, the data analysis focuses on the semantic and phonological relations and their interactions by lexical associations.

\subsubsection{Analysis of sounds}

The relation of sounds between the stimuli and the associates was analyzed by the phonological similarity. Only the disyllabic responses were transcribed as IPA symbols to compare with the stimuli. A response was marked to have a phonological relation with the stimulus if they shared identical segments including an initial consonant, a rhyme or a whole syllable. Example (1) illustrates a phonological relation with the similarity on the second onset between the stimulus and the response; (2) shows the shared first syllable as well as the second rhyme between the word pair.

(1) 回收 ‘to recycle' [xwej.sow] > 減少 ‘to reduce’ [tøjen.saw $]^{4}$

The response [t6jen.saw] has the identical initial consonant of the second syllable to the stimulus [xwej.sow]; therefore, this word pair is considered to share a phonological relation.

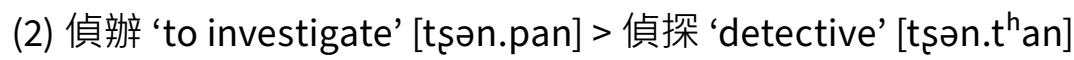

The response [tşn. $\mathrm{t}^{\mathrm{h}} \mathrm{an}$ ] has the same first syllable [tsən] and identical segments of the second syllable to the stimulus [tson.pan] including the nucleus [a] and the final

4 The dot (.) between IPA symbols indicates the syllable boundary. 
nasal [n]; therefore, the word pair shows the similarity of both initial (syllabic level) and rhyming (segmental) positions.

The identical initial consonants, rhymes, and syllables are included in the analysis of sounds based on the phonological similarity by a chi-square test from $3 \times 2$ tables to investigate the organization of the sound networks in Taiwan Mandarin ${ }^{5}$.

\subsubsection{Analysis of meanings}

The relation of meanings between the stimuli and the associates is illustrated by the classifications between syntagmatic relation and paradigmatic relation, which is also implemented by previous studies (e.g., Entwisle 1966; Nissen \& Henriksen 2006; Sheng et al. 2006). Word pairs are classified as representing a syntagmatic relation when the target and its response constitute a syntactic and semantic combination with different parts of speech, or share the same syntactic category with a clear sequential connection (e.g., bird and fly; accident and car). The paradigmatic relation on the other hand includes alternative semantic connections, such as synonymy, antonymy, hyponymy or meronymy, while targets which can be conventional or commonly regarded as a symbol are also classified to this type of semantic relation (e.g., coffee and tea; love and heart). The first example below (1a and $1 \mathrm{~b}$ ) demonstrates two types of syntagmatic relation; the paradigmatic relation is shown in (2). Several categories further proposed by Wan \& Ting (2019) are included in the classification, such as environmental relation, idiomatic relation, and nonrelation. The environmental relation indicates the interference to the response from personal linguistic experiences or the environment during the task, which is shown as example (3). The idiomatic relation refers to the idiomatic or conventional phrases in a language, as in (4). Nonrelation means no semantic relations between the word pair, as exemplified in (5).

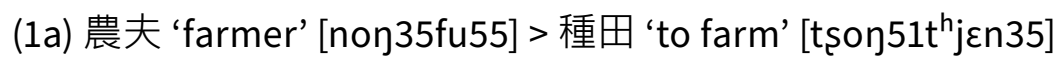

The stimulus 'farmer' has a syntactic connection to the response 'to farm', in which the first one is a noun whereas the other one is a verb. A farmer farms, so the two words are in a syntagmatic relation.

(1b) 太空 'outer space' [ $\mathrm{t}^{\mathrm{h}}$ aj51 $\mathrm{k}^{\mathrm{h}}$ on55] > 太空人 'astronaut' [ $\mathrm{t}^{\mathrm{h}}$ aj51k $\mathrm{k}^{\mathrm{h}}$ on55zən35]

Here is another example of the syntagmatic relation, in which even though the stimulus and the response share the same part of speech as nouns, there is a clear sequential connection between them for this classification.

5 The statistical analyses in the present study were computed by R (R Core Team, 2018). 
(2) 麵包 'bread' [mjen51paw55] > 麵粉 'flour' [mjen51fən21]

The stimulus 'bread' and the response 'flour' share the same superordinate in a 'bakery' setting, and can be placed in the same syntactic slot in a context; therefore, they are in a paradigmatic relation.

(3) 發明 'to invent' [fa55min35] > 豆芽菜 'bean sprouts' [tow51ja35tshaj51]

One of the participants associated the stimulus 'to invent' with his response as 'bean sprouts' because he had just watched a news programme about an invention related to bean sprouts. The association comes from the participant's personal experience, so it is an environmental relation.

(4) 模擬 'to imitate' [mo35ni21] > 兩可 'to be neutral' [ljan35k hr 21$]$

The response 'to imitate' to the stimulus 'to be neutral' is associated from a Mandarin Chinese idiom (Chengyu) 模稜兩可 'betwixt and between' [mo35 lən35 ljan35 $\mathrm{k}^{\mathrm{h}} \mathrm{r} 21$ ] to describe an ambiguous situation; therefore, it belongs to an idiomatic relation.

(5) 潛能 ‘potentiality’ [t ${ }^{h}$ jen35nəク35] > 漁夫 ‘fisherman’ [y35fu55]

The stimulus 'potentiality' and its response 'fisherman' do not show a semantic relation but are associated by the features encoded in the first character of the stimulus, which is attested by the participant.

Similarly, a chi-square value from $2 \mathrm{x} 2$ tables is evaluated with an effect size index reported to explore the general distribution between syntagmatic and paradigmatic relations in Taiwan Mandarin lexicon.

\subsubsection{Analysis within networks}

The analysis of the interaction between sounds and meanings applies Wan \& Ting's (2019) as well as Tang \& Wan's (2020) methodologies. The classification deals with either a semantic relation [+sem, -phon], phonological [-sem, +phon] relation, mixed relation [+sem, +phon], or nonrelation [-sem, -phon]. The semantic relation covers the syntactic as well as the paradigmatic relations shown as example (1). The phonological relation refers to the phonological similarity between the word pairs, i.e., shared initial consonants, rhymes or syllables, regardless of the syllable types, as in example (2). The mixed relation shares both the semantic connections and phonological similarities, and in example (3). Nonrelation means no semantic and phonological relations between the word pair shown as example (4). 


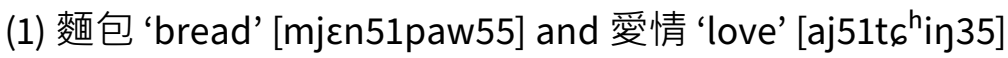

The stimulus 'bread' [mjen51paw55] and its response 'love' [aj51t6 ${ }^{\mathrm{h}}$ in35] have a semantic relation of the paradigmatic type due to an antonymic symbolization of physical and mental requirement respectively; however, they do not share any identical phonological segments with each other.

(2) 綠色 'green' [ly51sr51] and 過濾 'to filter' [kwo51ly51]

The response 'to filter' [kwo51ly51] is associated from the first syllable [ly] of the stimulus 'green' [1y51sr51] for its second syllable without any semantic relations, so they are in a $[-$ sem, +phon] relation.

(3) 扭曲 'to twist' [njow21t6 $\left.{ }^{\text {h}} y 55\right]$ and 彎曲 'to bend' [wan55t $\left.6^{\text {h}} y 55\right]$

The stimulus 'to twist' [njow21t $6^{\mathrm{h}} \mathrm{y} 55$ ] and its response 'to bend' [wan55t $6^{\mathrm{h}} \mathrm{y} 55$ ] are in a paradigmatic relation, and they also have an identical syllable [ $\mathrm{t} 6^{\mathrm{h}} \mathrm{y} 55$ ] for the phonological relation.

(4) 樹林 ‘woods’ [su51lin35] and 衣服 ‘clothes’ [i55fu35]

The response 'clothes' [i55 fu35] to the stimulus 'woods' [su51 lin35] is an association from a personal experience without any linguistically semantic connections or identical phonological segments shared between two words, so it results in a [-sem, -phon] relation.

\section{Associations between sounds and meanings}

A total of 248 responses from associations were collected from six Taiwan Mandarin native speakers, including nine monosyllabic words, 194 disyllabic words, and 45 words with more than disyllables, in which 160 nouns, 65 verbs, and two adjectives can refer the frequency values and parts of speech in Sinica Corpus.

\subsection{Networks of sounds}

Within the 248 responses, 194 disyllabic responses are included in the phonological analysis. In the analysis of the first syllable of the word pairs, seven initial consonants $(C)$, eleven rhymes (R), and ten syllables (S) are identical segments; in that of the second syllable, five initial consonants, fifteen rhymes, and ten syllables are marked as the shared segments as well. The chi-square result $X^{2}=0.8808(d f=2)$ does not reject the null hypothesis $(p=0.6438)$, indicating that the possibility for identical segments to present 
is not directly related to the syllable positions. The visualized distribution is presented as Figure 3.
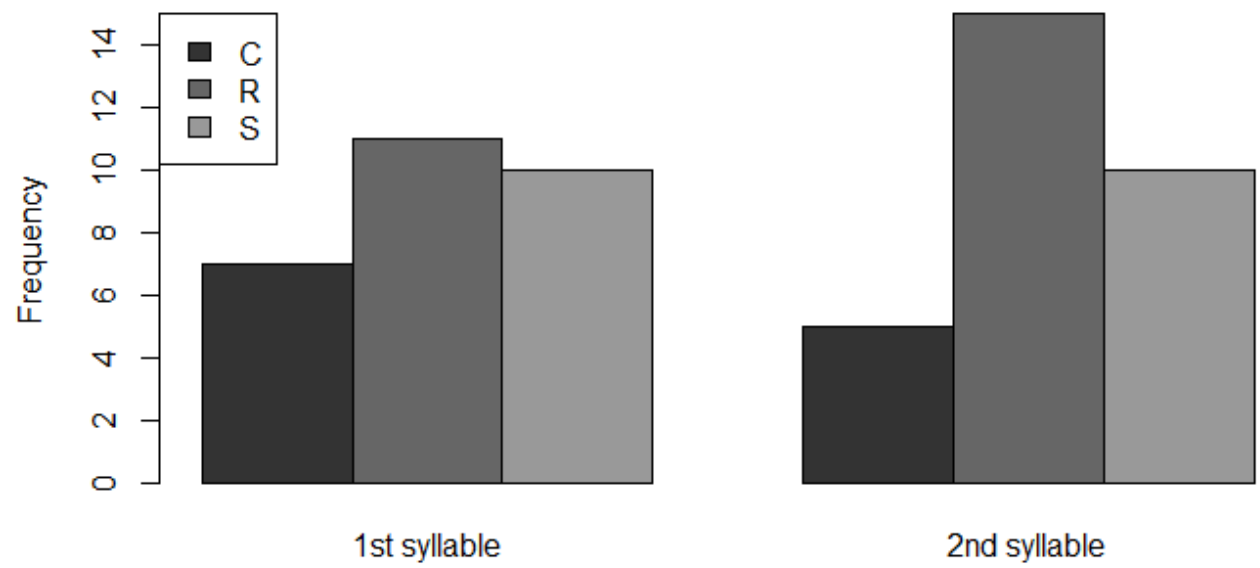

Stimuli

Figure 3. The frequency distribution of the phonological similarity of the responses

Only about $32.47 \%$ (63/194) of the responses are found with phonological similarity to the stimuli, either the initial consonants or the rhymes. Less identical initial consonants $(19.05 \%$; 12/63) present than the identical rhymes $(41.27 \% ; 26 / 63)$ do between the stimuli and the responses both in the first and second syllables. The identical syllables between the first syllable and the second one present a close distribution.

\subsection{Networks of meanings}

In this section, the 248 responses are analyzed regardless of the syllable types. They include: 126 responses with the paradigmatic relation $(\mathrm{P}), 108$ of those with the syntagmatic relation (S), seven with the environmental relation (E), two with the idiomatic relation (I), and five with no relation $(\mathrm{N})$ to the stimuli. Two main types of semantic relations, i.e., paradigmatic $(\mathrm{N}=126)$ and syntagmatic $(\mathrm{N}=108)$ relations, are further analyzed with syntactic categories of the stimuli to discover the relationship between these two variables. The distribution of the semantic relations based on the partsof-speech is analyzed by a chi-square test as well. The result $X^{2}=16.875(d f=1)$ rejects the null hypothesis $(p<.0001)$, indicating that the semantic relations are related to the syntactic categories of the stimuli during free lexical associations. The effect size index $\phi=.27$ shows that the syntactic categories have a near medium effect on the semantic relations according to a traditional criterion of a medium effect $(\phi=.30)$. The visualized statistics is presented as Figure 4. 

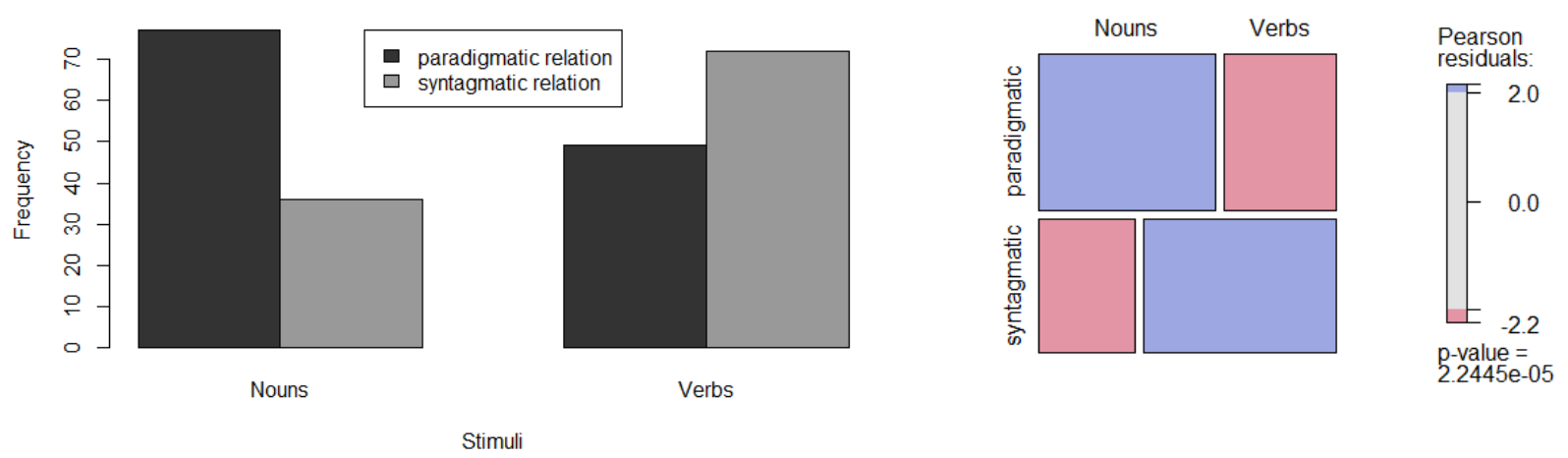

Figure 4. Bar plot and mosaic plot of the observed frequencies of the semantic relations

In the bar plot, the x-axis refers to the syntactic categories of the stimuli, the y-axis indicates the value of the frequency, and the grey bars show the number of the responses with the analyzed semantic relations. More noun responses share the paradigmatic relation with the noun stimuli and more verb responses share the syntagmatic relation with the noun stimuli. The mosaic plot represents the frequency by the square areas and residuals. The observed and the expected values are presented by colors.

Among 50.8\% (126/248) of the responses sharing the paradigmatic relation to the stimuli, $61.11 \%$ (77/126) and $38.89 \%$ (49/126) of them are responses to the nouns and verbs, respectively. On the other hand, 43.55\% (108/248) of the responses are found sharing the syntagmatic relation to the stimuli, and $33.33 \%$ (36/108) of them are responses to nouns, while $66.67 \%(72 / 108)$ of them are responses to verbs. The residuals indicate that the positive associations occur between nouns and the paradigmatic relation, as well as between verbs and the syntagmatic relation, while the negative associations present in the opposite situations.

\subsection{Between sounds and meanings}

Among the 248 responses, the semantic relation (+sem, -phon) has the highest proportion $(63.3 \%$; 157/248) among all the relations between sounds and meanings. The results also include eighty mixed relations (+sem, +phon), three phonological relations (-sem, + phon), and eight nonrelations (-sem, -phon). The visualized distribution of each type of relations is presented as Figure 5. 

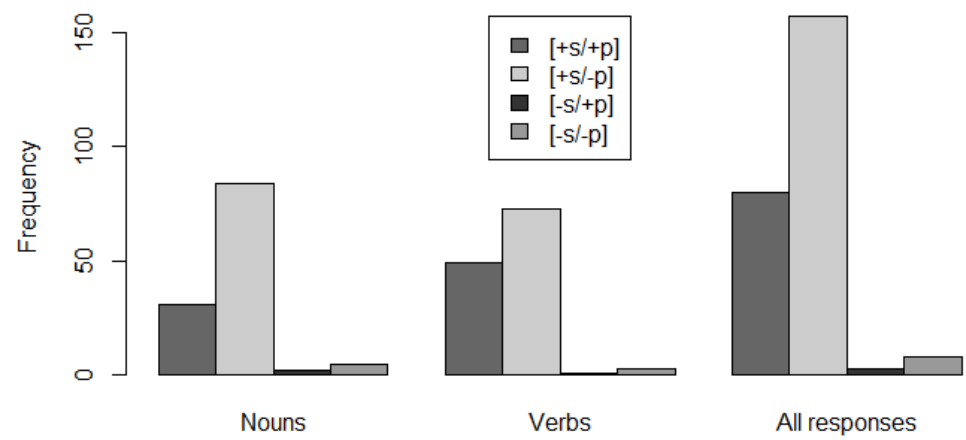

Figure 5. Bar plot of the frequency of the interaction between semantic and phonological relations of the responses

The results between the semantic and phonological relations indicate that the purely phonological relation of the [-sem, +phon] type accounts for $1.21 \%(3 / 248)$ of the data, the purely semantic relation of the [+sem, -phon] type accounts for $63.31 \%(157 / 248)$, and the mixed-type relation [+sem, +phon] accounts for $32.26 \%(80 / 248)$ of the data. Similar proportions can also be observed between the responses to the noun and verb stimuli respectively.

\section{Conclusion}

This study provides an insight into the lexical networks in Taiwan Mandarin by a free word association task. The unknown details embedded in each segment and connection signalled in the present work are undoubtedly worthy to continue the research. Previous works have already put much effort into the study of lexical access, retrieval, and organization in the mental lexicon; however, the related studies in Mandarin still leave a gap for the experimental basis of possible linguistic combinations. The features insufficiently considered by the previous works are especially phonological influences including initial consonants, syllable structures, and tones. Therefore, the word frequency, semantics, phonology, and parts of speech are all counterbalanced for the target words of lexical associations in the present study to fill the gap.

First of all, the possibility for identical segments to present in a syllable is not statistically proved by lexical associations, which may require a further examination since only 194 disyllabic responses are available for the analysis. However, the effect of rhymes is still found with a higher proportion between word pairs. As for the semantic relations, only a few of responses are marked as unrelated, and the consistency of the syntactic categories is generally stronger with responses having nouns as stimuli. This may also result in the higher proportion of the paradigmatic relation between the stimuli and the responses regarding that more nouns are produced. Similar results are shown 
in the previous research and the present study regarding semantic relations while the phonological networks may require a further study. Finally, the result of the interaction between semantics and phonology during associations indicates that a purely semantic relation is much more frequent than a purely phonological one. The finding suggests the semantic-oriented organization in the Taiwan Mandarin lexicon by associations as well as the different way to access the lexical item regarding the different results against the occurrence of speech errors.

Several insufficiencies of the present study should be mentioned and discussed here. Because of the small scale of the experiment with limited observations by few participants, the results may not authentically represent the majority. Therefore, an expansion of the research is required. Second, although the analyses are evidence-based, some of them, such as semantic and phonological relations, are determined without the exact example or evidence provided in the relevant literature. For instance, the interpretation of certain idiomatic or environmental usages during associations may involve a subjective viewpoint. Finally, further investigations contributing to phonological networks in Taiwan Mandarin or other tonal languages with larger research and experiments across languages seem to be worth undertaking.

\section{References}

Aitchison, J. 2012. Words in the Mind: An Introduction to the Mental Lexicon. Chichester: John Wiley \& Sons.

Beckage, N. M. \& Colunga, E. 2016. Language networks as models of cognition: Understanding cognition through language. In: A. Mehler, A. Lücking, S. Banisch, P. Blanchard \& B. Job (eds.), Towards a Theoretical Framework for Analyzing Complex Linguistic Networks, 3-28. Berlin: Springer.

Caplan, J. B. \& Madan, C. R. 2016. Word imageability enhances association-memory by increasing hippocampal engagement. Journal of Cognitive Neuroscience 28(10): 1522-1538.

Chang, Y. L., Wu, J. Y., Chen, H. C. \& Wu, C. L. 2016. The development of Chinese Radical Remote Associates Test. Psychological Testing (63) 1: 59-81.

Chinese Knowledge Information Processing Group (CKIP). Technical Report no. 93-05, Academia Sinica, Taiwan.

Chinese Knowledge Information Processing Group (CKIP). Extended-HowNet (E-HowNet) 2.0. Academia Sinica, Taiwan.

De Groot, A. M. 1989. Representational aspects of word imageability and word frequency as assessed through word association. Journal of Experimental Psychology: Learning, Memory, and Cognition 15(5): 824.

De Simone, F. \& Collina, S. 2016. The picture-word interference paradigm: Grammatical class effects in lexical production. Journal of Psycholinguistic Research 45(5): 1003-1019. 
Dell, G. S. 1985. Positive feedback in hierarchical connectionist models: Applications to language production. Cognitive Science 9(1): 3-23.

Dell, G. S. 1990. Effects of frequency and vocabulary type on phonological speech errors. Language and Cognitive Processes 5(4): 313-349.

Dell, G. S. \& O'Seaghdha, P. G. 1991. Mediated and convergent lexical priming in language production: A comment on Levelt et al. (1991). Psychological Review 98(4): 604-614.

Dell, G. S. \& O’Seaghdha, P. G. 1992. Stages of lexical access in language production. Cognition 42(1-3): 287-314.

Dell, G. S., Reed, K. D., Adams, D. R. \& Meyer, A. S. 2000. Speech errors, phonotactic constraints, and implicit learning: a study of the role of experience in language production. Journal of Experimental Psychology: Learning, Memory, and Cognition 26(6): 1355.

Duanmu, S. 1990. A formal study of syllable, tone, stress and domain in Chinese languages [Unpublished doctoral dissertation]. Massachusetts Institute of Technology.

Duanmu, S. 2000. The Phonology of Standard Chinese. Oxford: Oxford University Press.

Entwisle, D.R. 1966. Lexical Associations of Young Children. Baltimore: Johns Hopkins.

Greidanus, T. \& Nienhuis, L. 2001. Testing the quality of word knowledge in a second language by means of lexical associations: types of distractors and types of associations. The Modern Language Journal 85.4: 567-77.

Hsieh, S. K. 2006. Hanzi, concept and computation: A preliminary survey of Chinese characters as a knowledge resource in NLP [Unpublished doctoral dissertation]. Eberhard Karls Universität Tübingen.

Hsieh, S. K. 2016. Chinese semantics. In: Sin-Wai Chan (ed), The Routledge Encyclopedia of the Chinese Language.

Huang, C. R., Chen K. J., Chang, L. P. \& Hsu, H. L. 1995. An introduction to Academia Sinica Balanced Corpus. [In Chinese]. Proceedings of ROCLING VIII: 81-99.

Huang, C. R. \& Hsieh, S. K. 2015. Chinese lexical semantics. In: W. S-Y Wang \& Chaofen Sun (eds.), The Oxford Handbook of Chinese Linguistics. Oxford: Oxford University Press. Huang, P.S., Chen, H. C., Huang, H. C. \& Liu, C. H. 2009. The development of Divergent Thinking Test of Word Associative Strategy (DTTWAS). Psychological Testing 56(2): 153-177.

Kittredge, A. K., Dell, G. S., Verkuilen, J. \& Schwartz, M. F. 2008. Where is the effect of frequency in word production? Insights from aphasic picture-naming errors. Cognitive Neuropsychology 25(4): 463-492.

Lee, H. Y. 2014. The analysis of Chinese Word Remote Associates Test among high school students in Taiwan [Unpublished Master's Thesis]. National Taiwan Normal University, Taipei, Taiwan.

Lee, J. 2012. A study of word association tasks about the English-Chinese mental lexicon (in Chinese) [Unpublished Master's Thesis]. Xihua University, China. 
McClelland, J. L. \& Rumelhart, D. E. 1981. An interactive activation model of context effects in letter perception: I. An account of basic findings. Psychological Review 88(5): 375-407.

Mednick, S. 1962. The associative basis of the creative process. Psychological Review 69(3): 220-232.

Nissen, H. B. \& Henriksen, B. 2006. Word class influence on word association test results 1. International Journal of Applied Linguistics 16(3): 389-408.

Psychology Software Tools, Inc. [E-Prime 3.0]. 2016. Retrieved from https://www.pstnet. com.

Reeves, L. M., Hirsh-Pasek, K. \& Golinkoff, R. 1998. Words and meaning: From primitives to complex organization. Psycholinguistics 2: 157-226.

Team, R. C. 2018. R: A language and environment for statistical computing. R Foundation for Statistical Computing, Vienna.

Tang, M. \& Wan, I-Ping. 2020. Predicting speech errors in Mandarin based on word frequency. In: S. Qu \& W. Zhan (eds.), From Minimal Contrast to Meaning Construct, 289303. Singapore: Springer.

Vitevitch, M. S. 2008. What can graph theory tell us about word learning and lexical retrieval? Journal of Speech, Language, and Hearing Research 51(2): 408-422.

Vitevitch, M. S. \& Goldstein, R. 2014. Keywords in the mental lexicon. Journal of Memory and Language 73: 131-147.

Wan, I. P. \& Ting, J. 2019. Semantic relationships in Mandarin speech errors. Taiwan Journal of Linguistics 17. 2: 33-66.

Zhao, Y. 2013. Comparative research about the mental lexicon between international students and native speakers. [in Chinese: liu xue sheng yu mu yu zhe xin li ci hui de bi jiao yan jiu]. Science \& Technology Information 18: 62-63.

Zhang, J. \& Chen, D. 2018. An empirical study on Chinese college students' associative reaction to Chinese mental lexicon. Applied Linguistics 4: 75-84.

\section{$* * *$}

Tzu-Yi Tseng has recently accomplished her Master's degree of Art in Linguistics at National Chengchi University (NCCU), Taipei, Taiwan. She is currently the head assistant of the Phonetics and Psycholinguistics Laboratory of NCCU where she is responsible for the automatic phoneme-aligned spoken corpus construction in Taiwan Mandarin. Tzu-Yi Tseng also continues her psycholinguistic research about lexical organization in Taiwan Mandarin as well as sentimental analysis between tone languages and stress languages. 